\title{
A!
}

This is an electronic reprint of the original article.

This reprint may differ from the original in pagination and typographic detail.

Rekola, Heikki T.; Hakala, Tommi K.; Törmä, Païvi

\section{Lasing in small-sized aluminum nanoparticle arrays}

Published in:

Metamaterials, Metadevices, and Metasystems 2017

DOI:

$10.1117 / 12.2274332$

Published: 01/01/2017

Document Version

Publisher's PDF, also known as Version of record

Please cite the original version:

Rekola, H. T., Hakala, T. K., \& Törmä, P. (2017). Lasing in small-sized aluminum nanoparticle arrays. In N.

Engheta, M. A. Noginov, \& N. I. Zheludev (Eds.), Metamaterials, Metadevices, and Metasystems 2017 (pp. 1-7).

[103432E] (Proceedings of SPIE; Vol. 10343). SPIE. https://doi.org/10.1117/12.2274332

This material is protected by copyright and other intellectual property rights, and duplication or sale of all or part of any of the repository collections is not permitted, except that material may be duplicated by you for your research use or educational purposes in electronic or print form. You must obtain permission for any other use. Electronic or print copies may not be offered, whether for sale or otherwise to anyone who is not an authorised user. 


\section{Lasing in small-sized aluminum nanoparticle arrays}

Heikki T. Rekola, Tommi K. Hakala, Päivi Törmä

Heikki T. Rekola, Tommi K. Hakala, Päivi Törmä, "Lasing in small-sized aluminum nanoparticle arrays," Proc. SPIE 10343, Metamaterials, Metadevices, and Metasystems 2017, 103432E (24 August 2017); doi: $10.1117 / 12.2274332$

SPIE Event: SPIE Nanoscience + Engineering, 2017, San Diego, California, United States 


\title{
Lasing in small-sized aluminum nanoparticle arrays
}

\author{
Heikki T. Rekola ${ }^{a}$, Tommi K. Hakalaa , and Päivi Törmäa \\ ${ }^{a}$ COMP Centre of Excellence, Department of Applied Physics, Aalto University, FI-00076 \\ Aalto, Finland
}

\begin{abstract}
We study lasing in regular arrays made from aluminum nanoparticles. We show that these structures function as laser sources at visible wavelengths, even when scaled to an order of magnitude smaller areas compared to existing literature. The aluminum nanoparticles provide a robust platform for studying lasing in plasmonic systems, even when the optical losses are higher compared to silver or gold.
\end{abstract}

Keywords: Plasmonics, Nanoparticle array, Plasmonic laser, Surface lattice resonance, Aluminum nanoparticles

\section{INTRODUCTION}

For studying nanoscale lasing, plasmon resonances supported by metallic nanoparticles provide a platform to build from. The resonances are typically short-lived (in the order of $10 \mathrm{fs}$ ), and confine the optical fields to nanoscale field volumes. These two features push the operating conditions of the devices to a new regime, where speed and massive optical gain are basic requirements.

There has been two complementary approaches for achieving lasing with metallic nanoparticles as the resonators. One is to start with a single nanoparticle, surround it with the gain material, and try to get stimulated emission into the surface plasmon resonances of the particle. ${ }^{1,2}$ The high optical losses of a single nanoparticle limits the viability of this approach.

The second approach is to start with multiple nanoparticles, and couple them together as a metamaterial through the optical near-fields ${ }^{3,4}$ or as plasmonic lattices, ${ }^{5}$ where far-field radiation couples the particle resonances together. The lattice approach has proven successful both in the infra-red wavelengths, ${ }^{6-8}$ and at visible wavelengths. ${ }^{9,10}$ As the particles are coupled together, the mode volume becomes larger, and allow for more gain media to interact with the optical fields. The reduced confinement also increases the mode quality factor. For these reasons the requirements for achieving lasing are lower.

The existing body of work with plasmonic lattice lasers has focused on large area structures. Here we show that these large area structures can be scaled down and still function as laser sources, narrowing the gap between the single nanoparticle lasers and the nanoparticle array lasers. By surrounding the lasing arrays with mirror structures, we can reduce the radiative losses of the lasing mode. Together these results pave the way for even smaller nanoparticle array lasers, offering a practical route to study the different aspects of the system while progressing towards single nanoparticle lasers. Structures as small as $24 \mathrm{x} 24$ aluminum nanoparticles $(10 \mu \mathrm{m} \times$ $10 \mu \mathrm{m})$ are shown to function as laser sources, utilizing organic molecules in a liquid as the gain medium.

\section{RESULTS}

We fabricate regular arrays of aluminum nanoparticles on glass substrates using electron beam lithography. The sample structure is shown schematically in Fig. $1 \mathrm{a}$ and b. The particles are $100 \mathrm{~nm}$ in diameter and $50 \mathrm{~nm}$ thick, with interparticle spacings of $420 \mathrm{~nm}$ in both $\mathrm{x}$ and y directions. The size of the arrays is varied from 50 $\mu \mathrm{m} \times 50 \mu \mathrm{m}$ down to $10 \mu \mathrm{m} \times 10 \mu \mathrm{m}$ in $10 \mu \mathrm{m}$ steps. For the mirror structures we use $80 \mathrm{~nm}$ diameter aluminum nanoparticles with $210 \mathrm{~nm}$ interparticle spacing.

Further author information: (Send correspondence to H.T.R.)

H.T.R.: E-mail: heikki.rekola@gmail.com.

P.T.: E-mail: paivi.torma@aalto.fi, Telephone: +358 503826770

Metamaterials, Metadevices, and Metasystems 2017, edited by Nader Engheta,

Mikhail A. Noginov, Nikolay I. Zheludev, Proc. of SPIE Vol. 10343, 103432E

(C) 2017 SPIE · CCC code: 0277-786X/17/\$18 - doi: 10.1117/12.2274332

Proc. of SPIE Vol. 10343 103432E-1 
$\mathbf{a}$

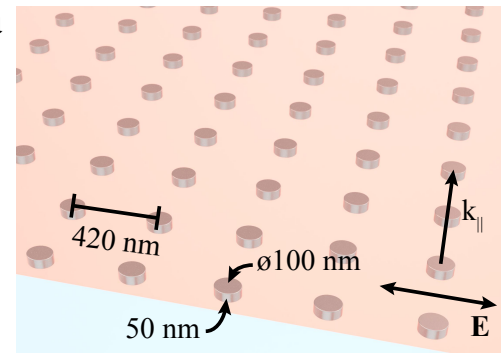

c

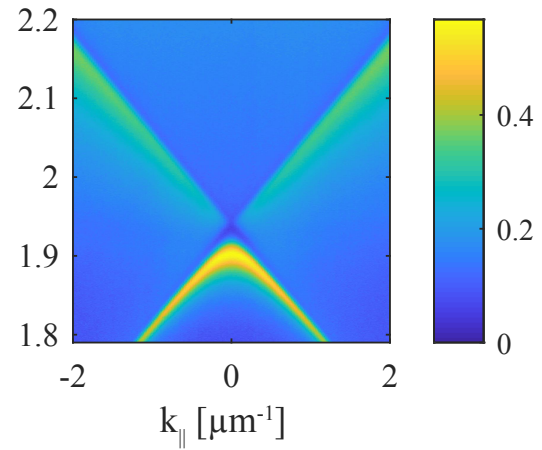

b

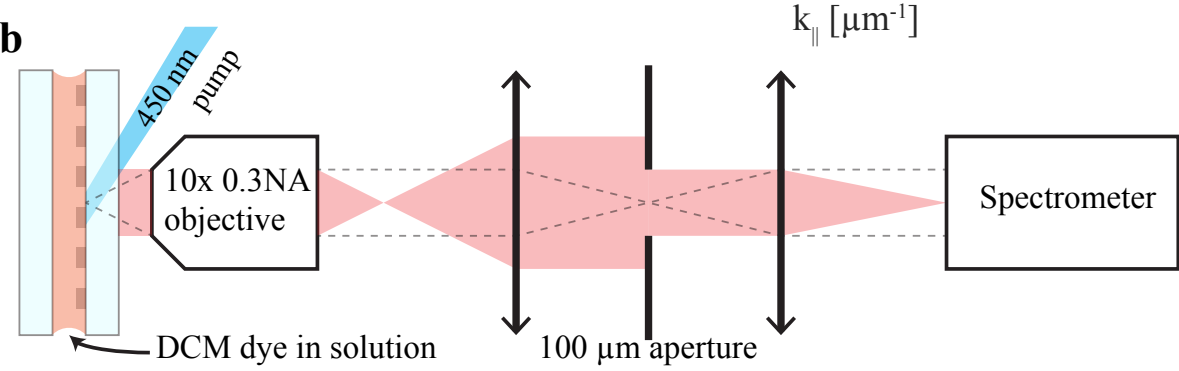

Figure 1. Schematic of the sample is shown in (a). The array periodicity $(420 \mathrm{~nm})$, particle diameter (100 nm) and height $(50 \mathrm{~nm})$ are highlighted, as are the electric field polarization with respect to $k_{\|}$, the direction where angle-resolved spectrum is obtained. The measurement setup is shown in (b). The array structures are on a glass substrate, covered with a drop of the DCM dye solution and an second glass slide. The dye molecules are pumped with $450 \mathrm{~nm}, 100 \mathrm{fs}$ pulsed laser from the side of the objective. The fluorescence emission from the molecules is collected with the objective, and the back focal plane is imaged to the center of the entrance slit of a spectrometer. A linear polarizer is used to select TE-polarized light, and a $540 \mathrm{~nm}$ long pass (not shown) is used to separate the fluorescence from scattered pump light. The measured angle-resolved extinction from a $420 \mathrm{~nm}$ period array, without dye molecules, is shown in (c). A white LED was used as the light source. The SLR modes are visible on top of a broad background coming from the spectrally wide surface plasmon resonance of the aluminum nanoparticles.

As the gain medium, a $6 \mathrm{ul}$ drop of $40 \mathrm{mM}$ 4-(Dicyanomethylene)-2-methyl-6-(4-dimethylaminostyryl)-4Hpyran (DCM) solution in a mixture of dimethyl sulfoxide and benzyl alcohol is sandwiched between the glass substrate and a second glass slide to form an approximately $6 \mu \mathrm{m}$ thick gain medium on top of the arrays. The refractive index of the solvent mixture ${ }^{11}$ is matched to the refractive index of the glass substrate.

The measurement setup is shown in Fig $1 \mathrm{~b}$. The samples are pumped with $450 \mathrm{~nm}, 100 \mathrm{fs}$ pulses at $1 \mathrm{kHz}$ repetition rate from an optical parametric oscillator. Fluorescence from the sample is collected with a 10X 0.3 NA objective (Nikon Plan Fluor). The pump spot on the sample is $300 \mu \mathrm{m}$ in diameter with flat intensity profile. The measured area is selected with an aperture to approximately $100 \mu \mathrm{m}$ in diameter. The back focal plane of the objective is imaged to the entrance slit of a spectrometer, allowing us to collect angle-resolved spectra from the sample structures.

The particle arrays support optical modes that form between diffracted waves in plane of the lattice, and the localized surface plasmon resonances (LSPRs). ${ }^{12}$ At normal incidence, the diffracted waves in plane of the lattice form a standing wave, providing the feedback necessary for lasing. The measured extinction of the 50 $\mu \mathrm{m} \times 50 \mu \mathrm{m}$ particle array for TE-polarization is shown in Fig $1 \mathrm{c}$. The array was covered with index matching oil to form a homogeneous refractive index environment. The crossing of two diffracted orders is visible in the extinction measurement. At the crossing point, the dispersion bends due to coupling of the two waves via the nanoparticle scatterers.

In Fig 2 a-c the angle resolved emission spectra of the $50 \mu \mathrm{m}$ array is shown below, at and above the lasing threshold. Below threshold (Fig 2 a) the dispersion of the SLR modes can be seen as spontaneous emission close to the nanoparticles can couple to the SLR modes, leading to directional emission. When the lasing threshold is reached (Fig $2 \mathrm{~b}$ ), emission intensity starts to increase in the SLR mode at $\mathrm{k}=0$. When the pump power is increased further, the SLR mode at $\mathrm{k}=0$ starts to dominate the emission spectrum. The laser emission spectrum 

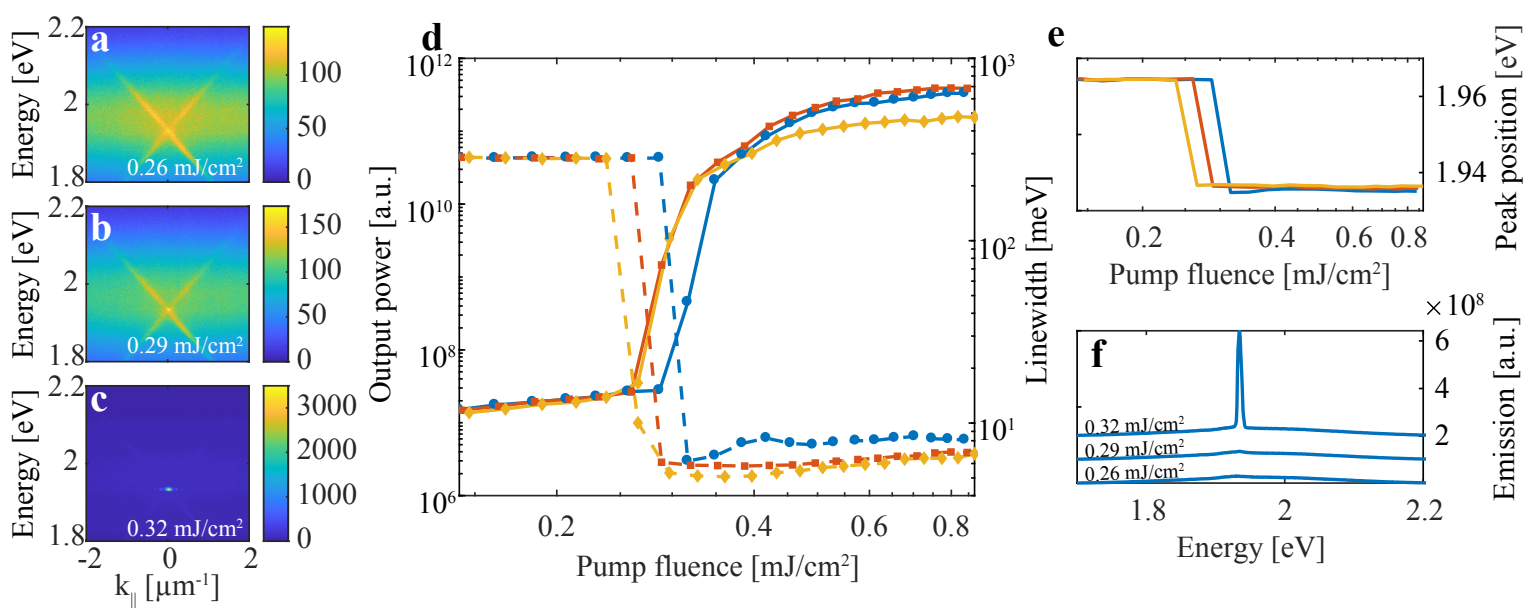

Figure 2. Lasing results for $50 \mu \mathrm{m}$ x $50 \mu \mathrm{m}$ sized arrays. In (a) - (c), the angle resolved emission patterns are shown below, at, and above the lasing threshold. The SLR dispersion is clearly visible in (a), while in (b) the SLR mode at $k_{\|}=0$ starts to gain more emission as the lasing threshold is reached. In (c), the SLR mode at $k_{\|}=0$ dominates the observed spectra. The dependence of the output power on the input power is shown in (d), together with the observed linewidth of the emission spectrum. Three different samples are compared, one without any peripheral structures (blue), one with $2 \mu \mathrm{m}$ wide mirrors surrounding the array (orange), and one with $10 \mu \mathrm{m}$ wide mirrors (yellow). A clear threshold can be seen in all cases, where the output power grows non-linearly and the linewidth drops down to 5 meV. The array structures surrounded with mirrors have slightly lower thresholds compared to the plain structure. The emission peak positions are shown in (e) for the three structures, with the same colors as in (d). The emission spectrum around $k_{\|}=0$ for the plain structure is shown in (f), with the pump powers matching (a)-(c). The lines have been offset vertically for clarity.

at $\mathrm{k}=0$ is shown in Fig $2 \mathrm{f}$, with pump powers corresponding to Fig 2 a-c. Below threshold, only the broad spontaneous emission from the DCM dye is seen. Above threshold, a narrow peak (limited by the spectrometer) is observed.

The emission intensities and linewidths for the lasing mode are shown in Fig $2 \mathrm{~d}$ for the $50 \mu \mathrm{m}$ array (blue), and for $50 \mu \mathrm{m}$ arrays surrounded by $2 \mu \mathrm{m}$ (orange) and $10 \mu \mathrm{m}$ (yellow) wide mirror structures. The addition of mirrors decreases the lasing threshold slightly, while the linewidth stays close to the resolution limit of the spectrometer used for the experiments. Curiously, the total emission power decreases for the $10 \mu \mathrm{m}$ mirror case, while the $2 \mu \mathrm{m}$ and no mirror cases are almost identical.

The observed maximum emission intensity location is shown in Fig 2 e. At the lasing threshold the observed emission maximum shifts in energy from $1.964 \mathrm{eV}$, which is the peak emission energy for the DCM dye, to 1.936 $\mathrm{eV}$.

Figure 3 a-c shows the angle-resolved emission below, at, and above the lasing threshold for the $30 \mu \mathrm{m}$ array. The SLR mode dispersion is less visible as a large amount of spontaneous emission is collected by the 100um aperture used to select the measured area. However, when the lasing threshold is reached in $3 \mathrm{~b}$, a single peak in energy is found centered at $\mathrm{k}=0$. Compared to the $50 \mu \mathrm{m}$ case shown in Fig 2, the lasing mode has a larger spread in $\mathrm{k}$, as can be expected for a physically smaller source. The emission intensity and linewidths are shown in Fig. $3 \mathrm{~d}$ for the array without mirrors, and with $2 \mu \mathrm{m}$ and $10 \mu \mathrm{m}$ mirrors. Compared to the $50 \mu \mathrm{m}$ arrays, the lasing threshold pump power is increased by $10 \%$. The peak position (Fig 3 e) and emission spectrum (Fig $3 \mathrm{f})$ are similar to the $50 \mu \mathrm{m}$ arrays.

When the array size is decreased further, to $10 \mu \mathrm{m} \times 10 \mu \mathrm{m}$, the effect of the array size becomes stronger. Figure $4 \mathrm{a}$-c show the angle-resolved emssion from the array below, at, and above threshold pump fluences. A wide spread in angle can be observed for the lasing peak, which still occurs at the energy of the SLR mode and is centered at $k=0$. Figure $4 \mathrm{~d}$ shows the emission output power and linewidths as a function of the pump power. The $10 \mu \mathrm{m}$ array without mirrors has a threshold of $0.76 \mathrm{~mJ} / \mathrm{cm}^{2}$, which is double compared to the 30 $\mu \mathrm{m}$ array. The effects of the mirrors surrounding the array structure become more pronounced as well, with the 

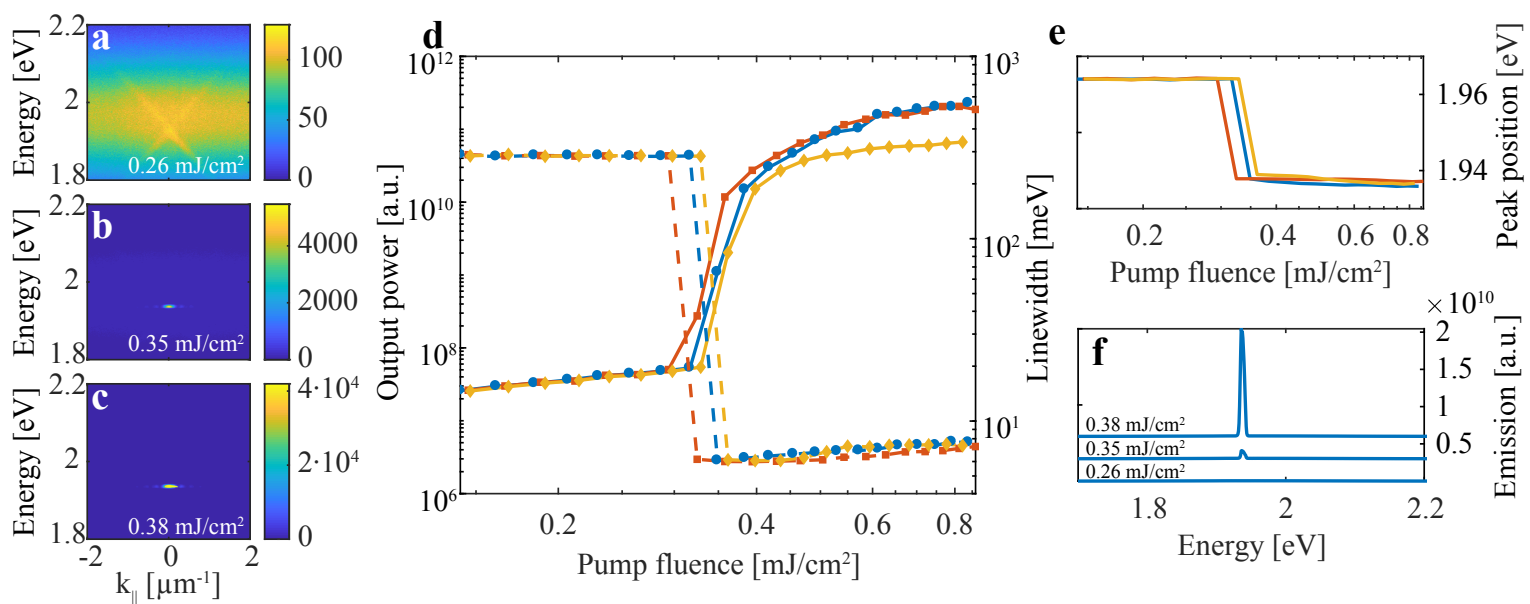

Figure 3. Lasing results for $30 \mu \mathrm{m}$ x $30 \mu \mathrm{m}$ sized arrays. In (a) - (c), the angle resolved emission patterns are shown below, at, and above the lasing threshold. The SLR dispersion is still visible in (a), while in (b) the SLR mode at $k_{\|}=0$ has already gained enough intensity to mask other features of the dispersion. In (c), the SLR mode at $k_{\|}=0$ is already slightly saturating the spectrometer CCD. The dependence of the output power on the input power is shown in (d), together with the observed linewidth of the emission spectrum. Three different samples are compared, one without any peripheral structures (blue), one with $2 \mu \mathrm{m}$ wide mirrors surrounding the array (orange), and one with $10 \mu \mathrm{m}$ wide mirrors (yellow). A clear threshold can be seen in all cases, where the output power grows non-linearly and the linewidth drops down to 6 $\mathrm{meV}$. The emission peak positions are shown in (e) for the three structures, with the same colors as in (d). The emission spectrum around $k_{\|}=0$ for the plain structure is shown in (f), with the pump powers matching (a)-(c). The lines have been offset vertically for clarity.

$2 \mu \mathrm{m}$ mirrors (14 $\mu \mathrm{m} \times 14 \mu \mathrm{m}$ total size) showing an order of magnitude more emission from the structure. The biggest difference comes in the threshold pump fluences - structure with $10 \mu \mathrm{m}$ mirrors $(30 \mu \mathrm{m} \mathrm{x} 30 \mu \mathrm{m}$ total size) has $28 \%$ lower threshold than the structure without mirrors. The peak positions shown in Fig 4 show that the $10 \mu \mathrm{m}$ mirror structure lases at a slightly $(2 \mathrm{meV})$ higher energy compared to the two other structures. The emission spectra in Fig $4 \mathrm{f}$ show an increased amount of spontaneous emission background, but above the lasing threshold they are similar to the $30 \mu \mathrm{m}$ and $50 \mu \mathrm{m}$ arrays.

The angle-resolved measurements of the arrays show multiple peaks at the lasing energy, symmetric with respect to the sample normal. These peaks are a sign of spatial coherence across the whole structure. If this is the case, the peak intensity and locations should match the single slit diffraction pattern intensity given by

$$
\left[\frac{\sin \left(k_{\|} D / 2\right)}{\left(k_{\|} D / 2\right)}\right]^{2}
$$

where $k_{\|}$is the in-plane momentum and $D$ is the array width.

Figure 5 shows the lasing emission angular dependence for the three arrays considered here as solid lines. The spontaneous emission background has been removed by subtracting the emission spectra just below threshold, and the main peak heights have been normalized to 1 . The curves are offset by 0.1 for clarity. The fitted single slit intensity patterns are shown as dashed lines. For the $10 \mu \mathrm{m}$ array the fitting gives an array size of $9.6 \mu \mathrm{m}$, for the $30 \mu \mathrm{m}$ the size is $28.4 \mu \mathrm{m}$ and for the $50 \mu \mathrm{m}$ case the fitting gives $44.8 \mu \mathrm{m}$. The slight discrepancy between the expected and fitted values can be due to non-homogeneous emission intensity across the array. Previously we have shown that the emission intensity maximized in the center of an array for lasing in the bright SLR mode, ${ }^{9}$ and this will favor slightly smaller values for the array size in the fitting process. 

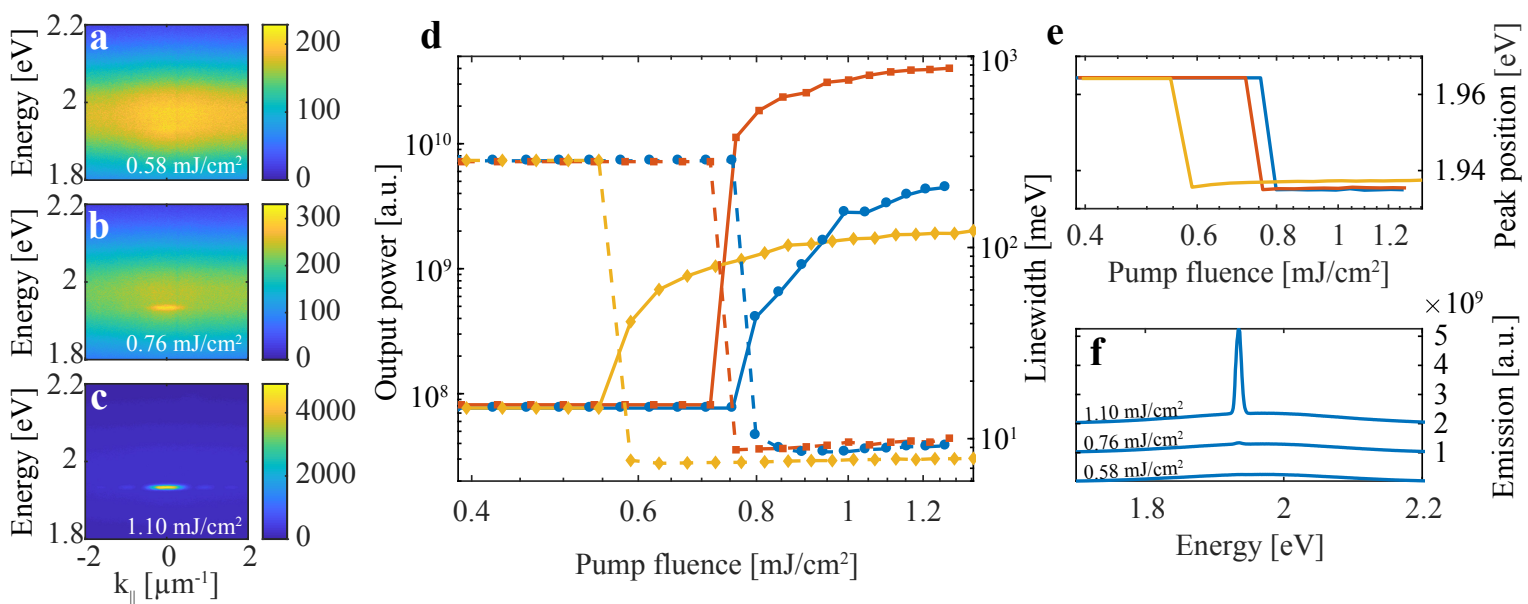

Figure 4. Lasing results for $10 \mu \mathrm{m}$ x $10 \mu \mathrm{m}$ sized arrays. In (a) - (c), the angle resolved emission patterns are shown below, at, and above the lasing threshold. The SLR dispersion is no longer visible above the spontaneous emission background. However, when the lasing threshold is reached, a peak appears at the same location as in Figs 2 and 3 . The dependence of the output power on the input power is shown in (d), together with the observed linewidth of the emission spectrum. Three different samples are compared, one without any peripheral structures (blue), one with $2 \mu$ m wide mirrors surrounding the array (orange), and one with $10 \mu \mathrm{m}$ wide mirrors (yellow). For these structures the effect of the mirror size becomes more pronounced. The $10 \mu \mathrm{m}$ mirrors show clearly the lowest threshold of the three structures compared, while the 2 $\mu \mathrm{m}$ mirrors show the most output power. The emission peak positions are shown in (e) for the three structures, with the same colors as in (d). The emission spectrum around $k_{\|}=0$ for the plain structure is shown in (f), with the pump powers matching (a)-(c). The lines have been offset vertically for clarity.

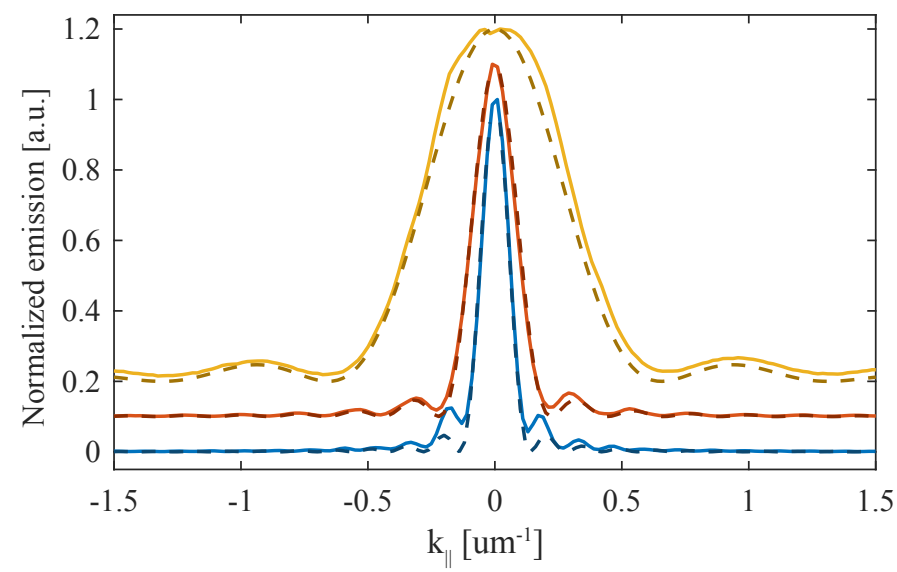

Figure 5. Emission pattern of the laser emission for arrays of $50 \mu \mathrm{m}$ x $50 \mu \mathrm{m}$ (blue solid line), $30 \mu \mathrm{m} \times \mu \mathrm{m} 30$ (orange solid line), and $10 \mu \mathrm{m} \times 10 \mu \mathrm{m}$ (yellow solid line) in size. The spontaneous emission background has been removed by subtracting the below threshold emission intensity from the data. The curves have been offset by 0.1 for clarity. The emission pattern shows several peaks, which fit very well to the single slit emission pattern given by Eq. 1 . The fitted emission patterns for array sizes of $44.8 \mu \mathrm{m}$ (blue dashed line), $28.4 \mu \mathrm{m}$ (orange dashed line) and $9.6 \mu \mathrm{m}$ (yellow dashed line) are shown on top of the measured data.

\section{DISCUSSION}

We have shown lasing in the optical modes of aluminum nanoparticle arrays. The devices show clear thresholds, with linewidths of the emission limited by the resolution of the spectrometer used. The emission is polarized, and spatially coherent across the structures.

The results with first-order structures as mirrors around the nanoparticle arrays show how the amount of radiative loss in-plane of the substrate can affect the device behavior significantly. The effect becomes stronger as the arrays are made smaller. This is to be expected, as the edges will play a larger role for small scale structures. 
With the $10 \mu \mathrm{m} \times 10 \mu \mathrm{m}$ arrays the addition of the mirrors structures enabled lower thresholds and increased output power. It remains an open question if the structures could be scaled down even more. Another open question is the relevance of the 2D structure with rectangular lattices; the two lattice directions are not strongly coupled, therefore could $1 \mathrm{D}$ chains, or narrow 2D array strips function as laser sources with even further reduced number of particles?

The aluminum nanoparticle arrays show promise as a platform to study lasing in plasmonic structures. The SLR modes with aluminum nanoparticles have lower quality factors compared to silver, but this can be overcome with high concentration of gain molecules. The main benefit of using aluminum instead of silver is it's robustness. Compared to our previous results with silver nanoparticles, ${ }^{9,13,14}$ the samples with aluminum stay functional for months, instead of a few days observed with silver. Higher pump laser intensities can be used. The increased robustness is due to two factors, increased adhesion to the glass substrate, and the protective oxide that forms on aluminum in contact with air. The absorption transition in the DCM gain molecules is also well matched with the LSPR resonance of the aluminum nanoparticles, enhancing the $450 \mathrm{~nm}$ pump laser absorption in the structures.

\section{ACKNOWLEDGMENTS}

This work was supported by the Academy of Finland through its Centres of Excellence Programme (2012-2017) and under project NOs. 284621, 303351 and 307419, and by the European Research Council (ERC-2013-AdG340748-CODE). Part of the research was performed at the Micronova Nanofabrication Centre, supported by Aalto University.

\section{REFERENCES}

[1] Stockman, M. I., "Spasers explained," Nat. Photonics 2, 327-329 (1 June 2008).

[2] Noginov, M. A., Zhu, G., Belgrave, A. M., Bakker, R., Shalaev, V. M., Narimanov, E. E., Stout, S., Herz, E., Suteewong, T., and Wiesner, U., "Demonstration of a spaser-based nanolaser," Nature 460, 1110-1112 (27 Aug. 2009).

[3] Zheludev N. I., Prosvirnin S. L., Papasimakis, N., and Fedotov V. A., "Lasing spaser," Nat. Photonics 2, 351-354 (June 2008).

[4] Chandrasekar, R., Wang, Z., Meng, X., Azzam, S. I., Shalaginov, M. Y., Lagutchev, A., Kim, Y. L., Wei, A., Kildishev, A. V., Boltasseva, A., and Shalaev, V. M., "Lasing action with gold nanorod hyperbolic metamaterials," ACS Photonics 4, 674-680 (15 Mar. 2017).

[5] Zou, S. and Schatz, G. C., "Narrow plasmonic/photonic extinction and scattering line shapes for one and two dimensional silver nanoparticle arrays," J. Chem. Phys. 121, 12606-12612 (13 Dec. 2004).

[6] Suh, J. Y., Kim, C. H., Zhou, W., Huntington, M. D., Co, D. T., Wasielewski, M. R., and Odom, T. W., "Plasmonic bowtie nanolaser arrays," Nano Lett. 12, 5769-5774 (14 Nov. 2012).

[7] Zhou, W., Dridi, M., Suh, J. Y., Kim, C. H., Co, D. T., Wasielewski, M. R., Schatz, G. C., and Odom, T. W., "Lasing action in strongly coupled plasmonic nanocavity arrays," Nat. Nanotechnol. 8, 506-511 (July 2013).

[8] Yang, A., Hoang, T. B., Dridi, M., Deeb, C., Mikkelsen, M. H., Schatz, G. C., and Odom, T. W., "Real-time tunable lasing from plasmonic nanocavity arrays," Nat. Commun. 6, 6939 (20 Apr. 2015).

[9] Hakala, T. K., Rekola, H. T., Väkeväinen, A. I., Martikainen, J.-P., Nečada, M., Moilanen, A. J., and Törmä, P., "Lasing in dark and bright modes of a finite-sized plasmonic lattice," Nat. Commun. 8, 13687 (3 Jan. 2017).

[10] Ramezani, M., Halpin, A., Fernández-Domínguez, A. I., Feist, J., Rodriguez, S. R.-K., Garcia-Vidal, F. J., and Rivas, J. G., "Plasmon-exciton-polariton lasing," Optica 4, 31-37 (20 Jan. 2017).

[11] Francesconi, R., Bigi, A., Rubini, K., and Comelli, F., "Excess enthalpies, heat capacities, densities, viscosities and refractive indices of dimethyl sulfoxide + three aryl alcohols at 308.15 K and atmospheric pressure," J. Chem. Eng. Data 50, 1932-1937 (1 Nov. 2005).

[12] Ross, M. B., Mirkin, C. A., and Schatz, G. C., "Optical properties of one-, two-, and Three-Dimensional arrays of plasmonic nanostructures," J. Phys. Chem. C 120, 816-830 (21 Jan. 2016). 
[13] Väkeväinen, A. I., Moerland, R. J., Rekola, H. T., Eskelinen, A.-P., Martikainen, J.-P., Kim, D.-H., and Törmä, P., "Plasmonic surface lattice resonances at the strong coupling regime," Nano Lett. 14, 1721-1727 (9 Apr. 2014).

[14] Shi, L., Hakala, T. K., Rekola, H. T., Martikainen, J.-P., Moerland, R. J., and Törmä, P., "Spatial coherence properties of organic molecules coupled to plasmonic surface lattice resonances in the weak and strong coupling regimes," Phys. Rev. Lett. 112, 153002 (14 Apr. 2014). 